\title{
Defining "Biodiversity"; Assessing Biodiversity ${ }^{1}$
}

\author{
Sahotra Sarkar, \\ Department of Philosophy, \\ University of Texas at Austin, \\ Austin, TX 78712.
}

\section{Introduction.}

The neologism biodiversity was coined by Walter G. Rosen at some point during the organization of the $21-24$ September 1986 National Forum on BioDiversity held in Washington, D. C., under the auspices of the US National Academy of Sciences and the Smithsonian Institution. ${ }^{2}$ The new term was intended as nothing more than a shorthand for biological diversity for use in internal paperwork during the organization of that forum. However, from its very birth it showed considerable promise of transcending its humble origins. By the time the proceedings of the forum were published (Wilson 1988), Rosen s neologism--though temporarily mutated as BioDiversity --had eliminated all rivals to emerge as the title of the book. The Washington forum was held only shortly after the founding of the US Society for Conservation Biology in 1985 which, sociologically, marked the formation of a new inter-disciplinary field dedicated to the conservation of biological diversity. That conference was almost immediately followed by the publication of Michael E.

\footnotetext{
1 Forthcoming in Monist. Much of what is said here emerged from years of collaboration and discussion with Chris Margules though he cannot be held responsible for any of the errors this contains. Our joint work is availabe as Sarkar and Margules (2001). This analysis was first presented as a seminar to the Unit for the History and Philosophy of Science, University of Sydney ([northern] Summer 1999). Comments by members of that audience, in particular Dan Faith and Paul Griffiths, were singularly helpful. For comments on previous drafts of this manuscript, thanks are due to Justin Garson, Bryan G. Norton, and Kim Sterelny.

2 Historical material about "biodiversity" is from Takacs (1996).
} 
Soul s (1985) manifesto for the new discipline, What Is Conservation Biology? which, by being published in BioScience, the journal with the widest general biological readership in the US, ensured an immediate high visibility for the emerging discipline. Within ecology, Daniel H. Janzen s (1986) exhortation to tropical ecologists to undertake the political activism necessary for conservation fortuitously appeared in 1986. A sociologically synergistic interaction between the use of biodiversity and the growth of conservation biology as a discipline occurred and it led to the reconfiguration of environmental studies that we see today: biodiversity conservation has emerged as the central focus of environmental concern.

The term biodiversity immediately found wide use following its invention. As Takacs has pointed out: In 1988, biodiversity did not appear as a keyword in Biological Abstracts, and biological diversity appeared once. In 1993, biodiversity appeared seventy-two times, and biological diversity nineteen times (1996, p. 39; italics as in the original). The first journal with biodiversity in its title, Canadian Biodiversity, appeared in 1991; a second, Tropical Biodiversity, appeared in 1992; Biodiversity Letters and Global Biodiversity followed in 1993. Meanwhile conservation biology as the science with the explicit goal of conserving biodiversity emerged as a highly visible enterprise with considerable political appeal in Europe and neoEurope. ${ }^{3}$ Primack (1993) published the first textbook of conservation biology in 1993; Meffe and Carroll (1994) followed with their comprehensive survey in 1994.

3 Essentially following Crosby (1986), "neo-Europe" is being used for places with a significant influx of, and political domination by, European settlers: for instance, large areas of the Americas, Australasia and southern Africa.

4 An adequate history of the emergence of conservation biology is yet to be written; see, however, Sarkar (1998b) for a sketch of that history. 
Nevertheless, the term biodiversity has remained remarkably vague and its measurement equally capricious. Is allelic diversity part of biodiversity? Or only species? What about individual differences? Do we have to worry about community structure? Is the number of species the appropriate measure? Do we have to take rarity and commonality into account? Or should we worry about differences between places? Both the problems of definition and measurement are widely acknowledged among conservation biologists though precious little is done about them. The purpose of this paper is an initial attempt to add enough precision to the concept of biodiversity to make its ordinal, if not quantitative, assessment plausible. This is done by returning the discussion of what biodiversity means from the abstract space in which it usually occurs to the definite historically contingent context in which the term emerged: biodiversity must be analyzed in the context of conservation biology and what it, as a goal-oriented enterprise that prescribes policies, must accomplish as it tries to conserve biodiversity.

Put bluntly, the position that this paper will argue for is that biodiversity is to be (implicitly) $^{5}$ defined as what is being conserved by the practice of conservation biology. Here, one distinction will be critical, and a medical analogy will help make that distinction. The analogy between conservation biology and medicine is deep and generally recognized. ${ }^{6}$ Both are goal-oriented enterprises and both embody norms that are structurally so similar that it seems natural to speak of ecosystem health, the maintenance of which is a central task of conservation biology. Both must operate

\footnotetext{
5 The sense of "implicit" here is the technical (axiomatic) one: a set of axioms implicitly define the concepts occurring in them. (Implicit definitions are to be contrasted with explicit definitions usually introduced by necessary and sufficient conditions.) In our case, however, we will have a variety of algorithms rather than axiom sets.

6 It was already mentioned in Soulé's (1985) manifesto.
} 
with whatever tools that are available, develop strategies to compensate for uncertain data and, quite often, untested models. Finally, just as medicine must adjust to the idiosyncracies of individual bodies, conservation biology must adjust to the peculiarities of places. This makes medicine different from, say, physiology; and conservation biology different from ecology. Those two latter disciplines try to obey the imperative of generalization that conventionally marks good science (though not necessarily always with success, especially in the case of ecology). ${ }^{7}$ Medicine and conservation biology are constrained from following that imperative over everything else: they must ensure that the peculiar individual entity that they are treating survives even if procedures unique to it have to be crafted. The medical analog of biodiversity is health, equally difficult to define explicitly, but implicitly embedded in the practice of good medicine.

In the medical context it is straightforward to make a distinction, though it may well only be a matter of degree, between ameliorative and preventive medicine. The former seeks to correct problems after they arise; the latter aims to prevent them through adequate precautionary measures: vaccinations, proper diet, and so on. What happens in an emergency room is an extreme case of ameliorative medicine. We practice conservation biology s analog of ameliorative medicine when, for instance, we allow a species to fall into decline and then make attempts at its recuperation. If we intervene only when it has regressed to the brink of extinction--as, for instance, in the United States, when we usually invoke the Endangered Species Act--, we are in the emergency room. Bringing a critically endangered species back from the brink of extinction is certainly part of maintaining biodiversity just as success in the emergency

7 For a critique of ecology that emphasizes this problem, see Shrader-Frechette and McCoy (1993). 
room is part of maintaining health. But just as a definition of health would be skewed if we were to rely solely on the measures we take in emergency rooms, or even (more generally) on ameliorative medicine, concentrating on endangered species (or other entities on the brink of extinction) will skew our definition of biodiversity.

Instead, we should focus on the preventive part of conservation biology. ${ }^{8}$ Since the task of conservation biology is to conserve biodiversity, conservation should be practiced even before population declines or other indicators of ecological trouble have appeared. From this point of view, devising preventive procedures is the central task of conservation biology. But how is this to be achieved? Over the last five years a unified and comprehensive framework for this task has emerged though--to the best of my knowledge--it has never been fully and explicitly presented before. The major pieces have been developed in Australia and the United States though contributions have also come from India, South Africa and the United Kingdom (and, quite possibly, other places). The basic slogan, and this is as yet no more than a slogan, is the adaptive management of landscapes.

The actual framework that has been developed is one for the prioritization of places for biodiversity value ${ }^{9}$ and the formulation of management procedures for the

8 In a philosophically very important paper Caughley (1994) distinguishes between a "small numbers paradigm" which, as he correctly notes, has dominated conservation biology in the US, and a "declining numbers paradigm" which, according to him, has been dominant in Australia. Implicit in Caughley's analysis is an only partly veiled criticism of the former: sheer stochasticity can take a population to extinction if its size has been allowed to fall low enough no matter what type of ameliorative intervention we then engage in. Population viability analysis (PVA), the theoretical technique developed primarily in the US to study the fate of such small populations (Boyce 1992), has amply demonstrated the difficulty of preventing the extinction of small populations. Caughley's point is that we should intervene as soon as declines set in, determine the ecological factors responsible for the declines, and counteract them. I go further to suggest that conservation biology be practiced even before the onset of declines.

9 This is to be distinguished from prioritization for biodiversity content (see below, in the text). 
long-term (in principle, infinite) survival of the biological units of interest. The entire process is supposed to be periodically iterated because species (or other units) may have become extinct--or have recovered from problems--in the interim, thereby changing the biodiversity value of a place, or because management practices may have turned out to be ineffective. This is the only sense in which the process is supposed to be adaptive. However, the framework is so new--it is yet to be fully implemented anywhere--that we have no idea whether this requirement of being adaptive will necessitate any change in the framework as it is currently understood. At present adaptive management is only a slogan embodying a tantalizing promissory note. However, at least the basic prioritization-management framework can be explicitly articulated. But, before I turn to that, a few points about place need to be noted.

Talk of place takes us into intuitively trivial but, strangely, philosophically relatively uncharted territory. A place is geographically rooted and loses its sense of place exactly as it is generalized about: a place is a specific region on Earth's surface filled with the particular results of its individual history. To generalize about a place involves abstraction from these particulars: the more we abstract, the more we lose the peculiarities that made it that place. But places precise biogeographic locations are what matter for conservation: they alone retain the heterogeneity that provides the intuition for biodiversity. A preference for a place is not merely a preference for an ecosystem or even a habitat, ${ }^{10}$ both of which are supposed to admit abstract characterization: the same habitat at different places may hold a different

\footnotetext{
10 Both "ecosystem" and "habitat" are unusually vague terms; "ecosystem" used to be generally understood in terms of an ecosystem ecology which used physical variables (most notably energy flows) to describe spatially extended biological systems. In that context, "habitat" was more rooted in place but habitats are also classified by type (wetland, tropical wet forest, and so on). These days the two terms seem to be used interchangeably.
} 
complement of genes, species, communities, or whatever other unit that may be of conservation interest. As noted before, worry about unique entities such as places takes us along a direction opposite to that of conventional scientific generalization. Philosophy--historically and, at least, the contemporary analytic tradition in Western philosophy ${ }^{11}$--has largely followed science in the deification of generality. Consequently places seem inappropriate loci of philosophical or scientific interest. Nevertheless, in the context of biodiversity conservation, we have to worry about the peculiarities of individual places, what entities they contain, what processes they admit, and what constrains those processes, all of which being subject to the contingencies of biological and geophysical history. As Leopold (1949, p. 196) put it in his inimitable way: One cannot study the physiology of Montana in the Amazon. Since, we have neither the economic nor the human resources to conserve every place of any biological interest--we would end up wanting to conserve Earth itself!-plans for conservation must ultimately involve a prioritization of places.

Returning, now to the prioritization-management framework, conserving biodiversity requires a (preferably periodically iterated--recall the remarks about adaptive above--) four-stage process:

(i) we must select some feature by which biodiversity may be estimated; such a feature is usually called a surrogate and the problem of choosing and estimating it is called the surrogacy problem. Surrogacy is a relation between an estimator parameter and a target parameter--this will be discussed below. Potential candidates as surrogates for biodiversity include the number of species or other biotic entities

\footnotetext{
11 See, in contrast, Malpas (1999). In general, the so-called continental tradition in Western philosophy has paid much more attention to "place" than the analytic one.
} 
( richness ), complexity of trophic webs, and so on. From what was said above, it should be obvious that the surrogacy problem is non-trivial, and much of this paper (SectionSection 2 -3) will be spent arguing for a particular type of solution. Suffice it here to note that whatever surrogates we finally choose must be ones that can be practicably assessed in the field;

(ii) once we have selected acceptable surrogates, we can use them to order places on the basis of their biodiversity content. This is the place prioritization problem. How we choose to solve it is critical to the definition of biodiversity. The major point being made in this paper (Section 4) is that each place prioritization algorithm implicitly defines a (slightly) different concept of biodiversity; thus, we are left with a family of (related) biodiversity concepts;

(iii) once we have a prioritized list of places on the basis of biodiversity content, we can proceed to assess the projected long-term future of entities of interest in them, for instance, populations of species. ${ }^{12}$ This is the viability problem. A variety of methods have been developed towards this end, for instance, stochastic population viability analysis (PVA) for small populations ${ }^{13}$ as well as more conventional ecological methods (Caughley and Gunn 1996). These methods provide estimates for a variety of relevant parameters, for instance, in the case of populations, the expected time to extinction and the probability of extinction within a specified time period, given the intrinsic growth rate, carrying capacity, and other ecological parameters. The biodiversity value of a place consists of its rank by both biodiversity content and an

\footnotetext{
12 Logically, we can attempt to solve the viability problem without place prioritization. However, in practice we would be unlikely to do so. Unless we were thinking of protecting some places and not others, there is little reason to attempt something as complicated as viability analysis.

13 See Boyce (1992) for a review.
} 
assessment of the viability of the entities of interest. Once we know the biodiversity value of places, we can establish a new prioritization using it;

(iv) finally, we are at the stage when a discussion of appropriate policies for the management of a place can begin. This is the feasibility problem. Socio-economic and political factors are typically more important in the formulation of such policies than purely scientific factors. People live in places and, by and large, have created them, that is, made them that place. Ignoring people--or, worse, excluding them--is why many conservation programs fail miserably, most notably Project Tiger in India. ${ }^{14}$

This paper is only about the first two stages of this process because, at the point when those have been successfully completed, the problems of defining biodiversity and assessing biodiversity are solved. Section 2 will show how complicated the former problem is. Section 3 is about the surrogacy problem; its solution will show how biodiversity should be assessed. Similarly, Section 4 is about the place prioritization problem; its solution provides a definition of biodiversity. Finally, Section 5 draws some conclusions.

14 A detailed history of Project Tiger is yet to be written; see, however, Guha (1989) and Guha and Martinez-Alier (1998). 


\section{The Problem of Biodiversity.}

What makes the definition of biodiversity difficult is that the biological realm-entities and processes--is marked by variability at every level of complexity. Restricting attention to entities, ${ }^{15}$ two different hierarchical schemes are standardly used for biological classification $^{16}$ : (i) a spatial (or generalized ecological) hierarchy starting from biological molecules and macromolecules, through cell organelles, cells, individuals, populations (demes) and meta-populations, communities, ecosystems (communities and their physical habitats) ultimately to the biosphere; and (ii) a taxonomic hierarchy from alleles through loci, linkage groups, genotypes, subspecies, species, genera, families, orders, classes, phyla and kingdoms.

(Many intermediate levels are ignored in this description.)

There are two points to note about either hierarchy: (i) it is not clean in the sense that biological entities fall into place in an exceptionless operationally welldefined fashion; (ii) there is heterogeneity, responsible for biological diversity, at every 15

Concern for processes leads to arguments for the conservation of biological "integrity" rather than diversity and is beyond the scope of this paper; for a critical discussion, see Karr (1991) and Angermeier and Karr (1994).

\section{6}

The philosophical importance of distinguishing between these two hierarchies is argued for in Sarkar (1998a) where it is shown that it can shed significant light on the units of selection controversy. It shows why alleles and individuals can simultaneously be units of selection contrary to the popular philosophical view that selection on one of these two units is in conflict with selection on the other. Here, it is similarly useful because it shows that focusing simultaneously on units of conservation in the two different hierarchies, for instance, on alleles and ecosystems need not be inconsistent with each other whereas focusing entirely on different units in the same ecosystem, for instance, species over alleles, is much more problematic. For a different perspective on biological hierarchies, but one that also emphasizes the importance of precisely defining and distinguishing between different hierarchies, see Eldredge (1985).

17

Presumably both hierarchies reflect evolutionary history and are constrained by evolutionary mechanisms. Since conservationist practice should take full cognizance of operative evolutionary processes, understanding the relationships between phylogeny and these two hierarchies is, in principle, critical to the design of conservation regimes. In practice we almost never know these relationships fully but must proceed anyway. I will, therefore, ignore this fundamental detail. 
level. The second point is almost trivial and a few examples will suffice to delineate its scope: almost any two populations even of the same subspecies will differ in their allelic profiles; except for some clonal organisms, almost any two individuals of the same species will have different genotypes; there are virtually no two identical ecological communities anywhere, and so on. The first point is equally important: while some entities such as organelles and cells are reasonably well-defined, examples such as fungi, symbionts and clonal organisms show that even individual is not always precisely defined. ${ }^{18}$

Asexual species are notoriously hard to define and even sexual species, usually defined by the ability to interbreed with fertile offspring, present problems. ${ }^{19}$ The most striking problem is the existence of ring species. In Britain, the herring gull (Larus argentatus) is easily distinguished--on morphological as well as reproductive grounds--as a separate species from the lesser black-backed gull (Larus fuscus). ${ }^{20}$ However, as we go east beginning with the Scandinavian countries, and continuing around the North Pole, we find different subspecies of the herring gull which can each interbreed with the one (geographically) preceding it. Ten such subspecies are found as we traverse Siberia, cross the Behring Straits, continue through Alaska and Canada. The terminal subspecies in Britain is Larus fuscus which does not breed with Larus argentatus. Thus, the usual definition of species turns out not to be transitive!

\footnotetext{
18 Individuality, as Buss (1987) has persuasively argued, is itself a gradually evolved phenomenon which, therefore, is a matter of degree. Consequently the porosity of the category "individual" is hardly surprising.

19

See Sokal and Crovello (1970), reprinted in Ereshefsky (1992).

20

Details are from Maynard Smith (1975, pp. 212 -213).
} 
Conserving biodiversity, and construing the term intuitively to refer to all the biological diversity that there is at every level of both hierarchies, amounts to saying that biodiversity refers to all biological entities. Biodiversity in effect becomes all of biology. Conservation would be an impractical proposal if biodiversity is construed in this way. The standard move at this stage is to suggest that three entities capture what is important about biodiversity: genes (alleles), species, and ecosystems. As a simplifying proposal in the face of intractable complexity, this convention has merit. If we conserve allelic heterogeneity completely, we take care of much of the diversity below the genotypic and individual levels of our two hierarchies. ${ }^{21}$ If we conserve all species, we do conserve all entities at higher levels of the taxonomic hierarchy though we may not conserve interspecific hybrids which, because of the leakage in our classificatory schema, may not qualify for conservation. If we conserve all ecosystems we may conserve many communities and so on though this is hard to gauge: the highly fashionable term ecosystem is about the worst-defined in the ecological literature.

Nevertheless, even this catholic proposal falls afoul of the diversity of biological phenomena and does so in a rather spectacular manner. The monarch butterfly, Danaus plexippus, has two migratory populations in North America. Beginning in late August, the eastern population migrates to Mexico for five months. These butterflies aggregate in millions in the high-altitude fir forests in the Sierra Transvolcanica, some $80 \mathrm{~km}$ west of Mexico City. There are nine other such over-wintering sites all within an area of $800 \mathrm{~km}^{2}$ on isolated mountain ranges between 2900 and $3400 \mathrm{~m}$.

\footnotetext{
21 Much, but not all, unless we endorse a global genetic reductionism, that is, we espouse the view that all biological features are, in some significant way, reducible to the genes. For arguments against this position, see Sarkar (1998).
} 
Throughout the winter they remain sexually inactive. Survivors migrate north, starting in late March, and lay eggs on milkweed (Asclepias sp.) along the Gulf Coast. These 8-month-old remigrants die but their offspring continue migrating north towards Canada. Two or three more generations are produced over the summer. By the end of summer, the last summer generation enters reproductive diapause and instinctively begins a southerly migration towards Mexico. The western population shows similar behavior, migrating to about 40 known overwintering sites in California. What is striking about this behavior in both populations is that the migratory instinct is hereditary and, yet, so specific.

In California some measures have been taken to protect overwintering sites but the sheer cost of real estate may result in only a very few of the sites getting the necessary protection. The future of the Mexican sites may be even more bleak. Though, until recently, the high-altitude fir forests of Mexico had been relatively spared from adverse anthropogenic effects, they now face at least six threats ${ }^{22}$ : (i) largescale legal and illegal logging for timber and firewood; (ii) village expansion up the mountains; (iii) increased use of fire to clear land; (iv) invasion of the forests by lepidopteran pests; (v) spraying of Bacillus thuringiensis, an organic pesticide, the effect of which on monarch butterflies is unknown; and (vi) increased tourism.

The disappearance of overwintering sites will not necessarily mean the extinction of monarch butterflies: there are numerous non-migratory tropical populations. However, what will disappear is the remarkable migratory behavior of the two populations discussed above which has come to be seen as an example of

22 See Brower and Malcolm (1991). 
endangered biological phenomena. ${ }^{23}$ Other examples include the seasonal migrations of wildebeest in Africa and the synchronous flowering of bamboo in India. While the former certainly are spectacular and in danger of disappearing because of the construction of fences along migratory paths, the latter is perhaps even more peculiar. $^{24}$ One bamboo species, Thrysostachys oliveri, flowered in Burma in 1891 and seeds were sent to Calcutta and Dehra Dun, about $1500 \mathrm{~km}$ apart. Clumps raised at both these places flowered simultaneously in 1940. In 1961 there was simultaneous flowering of Muli bamboo (Melocanna baccifera) in Assam and Dehra Dun, about $1500 \mathrm{~km}$ apart. In $1970-71$, there was simultaneous flowering of spiny bamboo (Bambusa arundinacea) throughout India after a lapse of 45 years. Clearly, a very precise biological clock exists in these species. In these cases, extinction of the species would also lead to the extinction of the phenomenon of synchronous flowering. More interesting, in this context, is that in extended habitats consisting of forests of a single bamboo species, flowering occurs in waves, starting at one end and ending up at the other. This is the phenomenon that would disappear if these habitats do, even if the species persisted elsewhere (for instance, as isolated stands in botanical gardens).

Protecting the holy trinity of genes, species and ecosystems will typically not save such phenomena. The examples given above clearly show why conserving species and, thus, the genes within them, will not be sufficient. If all the individual places at which these phenomena occur are conserved, it follows trivially that the phenomena will not disappear. However, sampling ecosystems and representing them may lead to the protection of other exemplars of the type in question. In that 23

See Brower's contribution to Meffe and Carroll (1994, pp. $104-106)$.

24

Details are from Bahadur (1986). 
case, even conserving representative ecosystems will not lead to the protection of these phenomena. The question is whether we can devise a definition of biodiversity that will lead to the protection of endangered and other biological phenomena of interest, along with all other components of biodiversity. The proposals put forth in this paper will not provide an adequate solution to this problem. 


\section{Surrogacy and the Assessment of Biodiversity.}

It turns out that defining biodiversity becomes easier once we have a handle on what sorts of biological entities and related parameters we have a chance of assessing in the field. At the very least, the discussion of the last section should have made it clear that, because of the breadth and accompanying imprecision in the meaning of biodiversity, no single parameter, whether or not it can be realistically estimated, is likely to capture all biological features that we may find of interest. We will at least partly have to settle on conventions that will never be fully satisfying. If a definition of biodiversity is to have any practical application, two problems must be solved: (i) a relatively theoretical one, what is to be measured?; and (ii) a practical

one, can we realistically obtain the data that we want? ${ }^{25}$ The former is the problem of quantification; the latter is the problem of estimation. Jointly, these two problems comprise the problem of assessing biodiversity. To solve both problems requires choices of surrogates.

To solve the quantification problem, we will have to use surrogates that serve as indicators of general biodiversity. Surrogacy is a relation between a surrogate or indicator variable and a target variable: the surrogate variable represents the target variable in the sense that it stands in for the target variable (that is, it replaces the target variable completely in all our subsequent considerations). The term surrogate has come to be used rather indiscriminately and I will introduce a distinction that will bring some order to the often confusing discussion of finding and measuring surrogates.

${ }^{25}$ See Williams and Humphreys (1994). 
This distinction is one between true surrogates and estimator-surrogates. True surrogates are supposed to represent general biodiversity, that is, the target variable is supposed to be general biodiversity. The only constraint on a true surrogate is that, in principle, it should be amenable to sufficient quantification to allow its estimation in the field, even if only with difficulty. Thus, once we decide on a true surrogate, we have solved the problem of quantification to the extent that we require. The trouble, of course, is that because we do not know what general biodiversity is, we will never be able to assert beyond controversy that we have found the true surrogate. Worse, empirical considerations alone will not allow a complete solution of this problem because of the indeterminacy of biodiversity: empirical arguments can only settle questions about relations between empirically wellspecified entities. Conventions will enter into any determination of true surrogacy and these conventions will have to be justified. The justification will be based on both practical and apparently theoretical considerations.

The decisive practical consideration will be that there will have to be a tractable estimator-surrogate for the true surrogate we choose: I will return to this issue shortly. Turning to the theoretical considerations, we are faced with the doubly unfortunate situation that there is a wide variety of candidates for true surrogacy and that justifications for any of them are theoretical only insofar as they are guided by deeplyheld theoretical intuitions. Consider four common and plausible candidates:

(i) character or trait diversity: the intuition behind this is that evolutionary mechanisms usually impinge directly on traits of individuals in populations (Williams and Humphreys 1996). The trouble is that trait is not a technical term within biology and trait diversity is, therefore, not precise enough to solve the quantification problem adequately; 
(ii) species diversity: as we shall see below, this can be made sufficiently precise. Moreover, species diversity is the measure in most common use in almost all practical discussions of biodiversity. However, this may well be because it is regarded as an adequate estimator-surrogate (see below) rather than as a true surrogate. Nevertheless, it is important to note that species are the most well-defined category above the genotype in the taxonomic hierarchy and, because of that, the intuition that species diversity is a good true surrogate has some theoretical merit. There are two problems: (a) compiling a list of all species--including microbial ones--for any region is expensive and time-consuming; and, moreover, (b) we know very well that there is much more to biodiversity than species diversity;

(iii) life zone diversity: this reflects the intuitions that (a) what is important is the variety of biotic communities with their associated patterns of interactions; and (b) that focusing on communities will ipso facto take care of species since communities are composed of species. The chief disadvantage is that, at least on the surface, the quantification problem seems intractable: any classification of communities seems to involve arbitrary conventions. Life zone classification provides a partial way out: it involves coupling the characteristics of some communities in a place, in particular vegetation, with environmental parameters such as elevation, precipitation and temperature (and, sometimes, soil types). For many areas of the world, fairly precise life zone classifications exist, for instance, the Holdridge (1967) classification for central America and Ohsawa (1987) for the Bhutan Himalaya. Because of this, for many regions, the quantification problem has a reasonably satisfactory solution so long as we make sure that our choice of life zones is suitably fine-grained, that is, the definitions are not so broad that many different localized patterns of biodiversity all fall under the same life zone. Should our choice be too coarse grained, a choice of some 
representation of a life zone will not ensure the full--or even an adequate-representation of all the biodiversity within that life zone. Nevertheless, there remains the nagging worry that even after paying due attention to this problem, life zone diversity does not fully take into account species--or even community--diversity simply because only a few communities are used to define life zones;

(iv) environmental parameter diversity: the most theoretically-justified intuition behind this is that each point of the space spanned by environmental parameters is a putative niche to be occupied by some species. Diversity of environmental parameters is also defined well enough to solve the quantification problem adequately. Note that since we would sample the environmental space uniformly, we do not fall afoul of the problem that niches are not defined independently of organisms. However, not all putative niches will be occupied, and the correlation between biodiversity in the sense of diversity somehow associated with living organisms and environmental parameters may not be very good. Again, since we cannot measure biodiversity directly, we also cannot estimate quantitatively how good this correlation is. Finally, when we select places, if we do so using environmental parameters on large enough spatial scales, we also run into the danger of losing convergent but different species and other biological units, for instance, marsupial analogs of placentals if we fail to include Australian places because we selected similar environmental parameter sets elsewhere.

There is no fully non-conventional solution to the true surrogacy problem. To choose any of these four candidates or any other will require the use of pragmatic criteria, in other words, the adoption of some convention. Two of our candidates have obvious disadvantages: as noted before, character diversity is too imprecise. It cannot be quantified even to the extent required to judge the adequacy of estimator- 
surrogates for it; environmental parameter diversity, besides having the other problems mentioned above, is also intuitively unappealing simply because it is manifestly non-biological--at best it is a plausible candidate for estimator-surrogacy. Our other two candidates--species and life zone diversity--remain plausible. Note that nothing requires us to have only one true surrogate and it seems reasonable to use both. Moreover, none of the candidates addresses the issue of endangered biological phenomena. This can simply be added to the other two. This seems ad hoc, and there seems to be no way out. It makes more sense to try to protect places that are critical for the persistence of endangered biological phenomena while prioritizing places (as described in Section 4).

In contrast to true surrogates, estimator-surrogates have a true surrogate as the target variable. For estimator-surrogates, it is critical that they can be rapidly and easily estimated. Since whichever true surrogate we choose will have to be reasonably precisely demarcated, even if only by convention, the estimator surrogacy relation is quantitatively precise. Moreover and this is a point that cannot be overemphasized it is an empirical relation that must be investigated through field work. ${ }^{26}$ What has to be shown is that there is a good--preferably (but unachievably) perfect--method to be predict true surrogate diversity using the estimator-surrogates.

Diversity (of true or estimator-surrogates) can be plausibly quantified in a variety of ways. MacArthur (1965) showed how the Shannon-Weaver measure of information (see Shannon [1948]) can be used to quantify species diversity. Roughly, this measures the variability within a community and captures intuitions such as that

\footnotetext{
26 This point is correctly emphasized in an important paper by Landres, Verner and Thomas (1988). In their terminology, estimator-surrogates are "indicators"; they also do not make the distinction between the two kinds of surrogacy that is introduced here.
} 
a community $X$ with $50 \%$ species $A$ and $50 \%$ species $B$ is more diverse than a community $Y$ with $90 \% A$ and $10 \% B$. It is trivial to extend this definition to life zones (though not to endangered biological or other phenomena). If this is the measure we adopt, then it must be shown that our estimator-surrogates allow us to predict it correctly. The strategy suggested here is different. Recall the discussion in Section 1 of how places are supposed to be prioritized on the basis of biodiversity content. Suppose that this is done for a small subset of spaces using the true surrogates. ${ }^{27}$ Now, repeat the prioritization using the estimator-surrogates. If the estimatorsurrogates are adequate, the results should be the same, and we can proceed to use estimator-surrogates in the future. ${ }^{28}$

Traditionally, the estimator-surrogate that has been the most popular is species richness, the number of species at a place, which is distinct from species diversity. (Returning to the example from the last paragraph, communities $X$ and $Y$ have the same species richness [namely, 2] though $X$ is more diverse than $Y$.) Gaston (1996) lists five lines of evidence that point to species richness as a good estimator-surrogate for biodiversity: (i) species richness can be correlated to many measures of ecological diversity and it functions better in this way than most estimator-surrogates including species diversity (see, also, Magurran [1988]); (ii) species richness is sometimes positively correlated with the number of higher taxa such as genera; (iii) when species richness is relatively high, it is correlated with trait richness; (iv) though this remains controversial, some parameters that are supposed to measure the complexity of community webs (including the number of edges and

27 It can practicably only be done for a small subset of places simply because the assessment of true surrogates is typically difficult.

28

Section 4 will show how this procedure implicitly uses the concept(s) of biodiversity as defined there. 
the length of directed paths along trophic levels) seem to be correlated with species richness; (v) relatively high species richness is also correlated with increasing topographic diversity. Of these five points, the second and third are irrelevant since they refer to richness at the level of higher taxa or traits rather than diversity; the third assumes that complexity of trophic webs is related to diversity which is unproven in the field; and the first and fifth are relevant only if we accept environmental diversity as a true surrogate, an option that was argued against above.

There are, moreover, two strong arguments against using species richness or, for that matter, richness of any estimator-surrogates to prioritize places: (i) it is wellestablished that prioritizing places by richness is usually not--according to current (empirical) data an effective way of targeting diversity. The top two (or more) places may be rich in the targeted surrogates but may have very similar surrogates. Conserving both may add nothing or very little to what would be obtained by conserving one and some other place. Conserving both would thus be an ineffective allocation of limited resources; and (ii) the reason why selecting places on the basis of richness is ineffective is that, even intuitively, richness is not the same as diversity. Diversity, including biodiversity, connotes difference and unlikeness the amount of variety. ${ }^{29}$ Finally, Gaston s analysis ignored spatial scales and it is implausible that his five claims hold at all scales.

There are at least seven more plausible candidates for estimator-surrogates:

\footnotetext{
29 Using richness is often connected with the identification and use of "hot-spots," that is, places with very high richness to prioritize places. Therefore, rejecting richness means rejecting such hotspot analysis. Note, however, that this is not the only use of "hot-spot." Myers $(1988,1990)$ and others have taken account of endemism, besides richness, in their definition of "hot-spot." These analyses come closer to the one in the text than those which are only based on richness.
} 
(i) environmental parameter composition ${ }^{30}$, that is, a list of parameters such as average temperature and rainfall, which are often easy to obtain. They are widely recorded or can be inferred from reliable models;

(ii) soil type composition which will be based on a classification of soil types using their composition and other physical properties. Once again such data are easily obtainable, and can sometimes be inferred from satellite images;

(iii) dominant vegetation composition which can also be inferred from satellite images:

Usually these three are used simultaneously. They have the obvious advantage of being relatively easily assessed. Thus the estimation problem has a good solution. However, the empirical question of the relation of these data to species diversity (let alone other potential true surrogates) remains unresolved. This question is being systematically investigated in New South Wales and Texas (and, very possibly, other places); Returning to the candidates for estimator-surrogacy, the other four are:

(iv) species composition, a list of the species occurring at a place. In practice we never have a complete list. Microscopic species are almost impossible to survey adequately and, usually, not even all larger species are surveyed;

(v) life zone composition, a list of the habitats occurring at a place (for instance, tropical wet forest, moist forest, etc.) based on a pre-determined classification. As noted above, classifications of this sort now exist for many areas of the world;

(vi) genus or other higher taxon composition, which is what we often have instead of composition at the species level;

30 These "compositions" should be viewed as a list of entries for each place. The importance of maintaining--and not compounding--such lists will be explained in Section 4. 
(vii) subsets of species composition, once again, what we may have to use because complete species compositions are almost never available in practice. For some taxa such as birds and butterflies, extensive information exists.

All four of these are the ones most commonly used in practice; but there has been very little empirical work establishing the adequacy of the last two as predictors of any true surrogate diversity. If species diversity is taken to be the (only) true surrogate, the adequacy of the first of these four (species composition) is obviously trivially guaranteed; for any of the other true surrogates the problem of determining its empirical surrogacy remains. An analogous point can be made about life zone diversity as the only true surrogate and life zone composition as an estimatorsurrogate for it. Moreover, trying to use total species composition as an estimatorsurrogate is unjustified for the simple reason that it cannot be assessed rapidly and easily, as is required for such surrogates. There is no reason to believe that the same estimator-surrogates will be the best one for all regions. But that is not necessary so long as the ones that are used are shown to be adequate for the context of their use. 


\section{Place Prioritization and the Definition of Biodiversity.}

Once true surrogates have been chosen (partly by convention), and the adequacy of a set of estimator-surrogates has been established empirically, the estimator-surrogates must be estimated in the field. In the interest of brevity, from this point on in this paper, unless explicitly indicated otherwise, surrogate will be used only to refer to estimator-surrogates. At this stage what we have in hand are a set of places and list of surrogates at each place. We now have to prioritize these places and the prioritization procedure will implicitly define what biodiversity is taken to be. But, to prioritize places in this fashion and this is a critical point we need no absolute concept or measure of biodiversity. We merely need to be able to decide whether place $A$ has greater biodiversity than place $B$.

If we accept this limited requirement to capture the intuition of biodiversity that is relevant for our purposes, it becomes fairly straightforward to give an operationally precise definition of biodiversity as a relative concept. Biodiversity will be relativized in two ways: (i) the definition will only try to say if place $A$ has higher (or the same or lower) biodiversity than $B$; and (ii) it will do so only against a background set of places $\Pi$. What our procedure (or algorithm) must do is this: given $\Pi$ and a set of new places, $\Sigma$, and the algorithm must prioritize these places on the basis of biodiversity using the surrogate lists for $\Pi$ and for each place in $\Sigma$. This will be done by considering two places at each stage and iterating the process over the entire set of places, $\Sigma$, resulting, finally, in a prioritized list.

Consider any pair of places $A$ and $B$. At the first step of the algorithm $A$ has higher (same/ lower) priority than $B$ relative to $\Pi$ if and only if its surrogate list has more (less/ the same number of) entries not in the list of $\Pi$ than the surrogate list of 
$B .^{31}$ This rule of prioritizing places by considering the number of new surrogates is called complementarity in the literature. ${ }^{32}$ Note that at this stage there may be a tie between $A$ and $B$. The way we choose to break such ties generates (slightly) different algorithms. We may break it using a random process (for instance, appealing to a random number generator or, what amounts to the same thing, using lexical order, that is, selecting whichever of $A$ or $B$ that is first on our list of places). This is a pure complementarity algorithm. Or, before invoking randomness, we may use rarity: we would give higher priority to the place which has more rare surrogates (either among those not already represented in $\Pi$ or in the entire data set). This choice generates complementarity-rarity algorithms. (In some versions of this algorithm, rarity is used before complementarity, resulting in "rarity-complementarity" algorithms. ${ }^{33}$ ) If we still have a tie after invoking rarity, we may introduce various other rules: adjacency (is a new place adjacent to one already in $\Pi$ ? If so, choose it because it will lead to larger connected reserves); area (choose smaller/larger places); cost (choose the place with the lower cost); even richness; and so on. Each combination and subsequent permutation generates a different algorithm.

31 In practice, the algorithms that are used are slightly more complex. Instead of only inquiring which surrogates do not occur in $\Pi$, they inquire which surrogates are not yet adequately represented in $\Pi$. However, the level of representation that is considered to be adequate is ultimately set by convention though it presumably will be guided by a fair amount of information about probabilities of extinction of different entities.

32 This principle was independently discovered at least four times: Kirkpatrick (1983), Ackery and Vane-Wright (1984), Margules, Nicholls and Pressey (1988) and Rebelo and Siegfried (1990). The clearest early statement is due to Margules, Nicholls and Pressey (1988); the term "complementarity" was introduced by Vane-Wright, Humphries and Williams (1991).

33 Rarity-complementarity algorithms are often supposed to be address the problem of ensuring the protection of endemic species (or other surrogates) better than complementarity-rarity algorithms (Aggarwal et al. 2000). While this is intuitively plausible, it has not been empirically demonstrated. 
Once a tie is broken, we update $\Pi$ by adding the selected place to it, and iterate the process. We stop the process once every surrogate is represented ${ }^{34}$ and the priority list consists of those places added to $\Pi$ in the order in which they were added. At the end, we should add places that are necessary for the persistence of endangered biological phenomena. Once again this seems ad hoc but no better solution seems to be forthcoming.

This iterative procedure shows how additions may be generated to a system of reserves. However, to set the iteration going, we need to specify a base-line: how $\Pi$ is to be constructed from the null set. In practice we will rarely face this problem: existing networks of protected areas will provide the reference set though, usually, because these places have traditionally been selected using criteria other than biodiversity (sublime landscapes, wildernesses, etc.), such reference sets will not be repositories of high biodiversity. Nevertheless, for the sake of definitional completeness, we must be able to solve the problem of definition the reference set ab initio. Consistent with the intuition that has guided our algorithms, we do so by invoking rarity. The first place(s) to be included in $\Pi$ are those that have the rarest surrogates. If there are places with surrogates that occur nowhere else, then these enter $\Pi$ at the beginning. If not, we choose the place(s) with the most rarest surrogates that are present in our data. 35

\footnotetext{
34 Usually (see Footnote 31) we wait until every surrogate is represented to the level of adequacy we initially chose.

35 An alternative way of initializing the process is to use richness (Kirkpatrick 1983). This is the only situation in which richness is sometimes more efficient than rarity in the sense that a smaller set of places may eventually suffice to ensure adequate representation of surrogates. However, the differences are not great.
} 
Different concepts of biodiversity are implicitly defined by these algorithms: biodiversity is the relation used to prioritize places. The crucial rule is complementarity: by focusing on new surrogates it naturally captures our intuition of biodiversity. With some rhetorical flourish we may just as well say that what matters is complementarity: the concept of biodiversity is parasitic on the concept of complementarity. That complementarity and rarity should have some role in our concept of biodiversity should not be controversial. Recall that our context is one of biological conservation. We want to protect what is threatened by extinction: by and large, rarity captures that intuition. ${ }^{36}$ We want to protect as many different entities that are not already adequately protected: complementarity exactly captures that intuition.

It is far less clear that we are still defining biodiversity when we invoke the other rules such as adjacency, area, or cost in our algorithms. I leave it as an open question whether we should restrict biodiversity to what is defined by algorithms that only invoke rarity and complementarity or whether we should be more catholic in our taste. I will adopt the latter approach in Section 5 though only for expository convenience.

Finally, it is important to note two points about these algorithms: (i) they operate on lists of surrogates for each space. We cannot compound these lists into single numbers--such as richness or MacArthur s diversity index--and continue to use these algorithms. Field work generally involves compiling such lists. Yet, it is common practice among field biologists to reduce these lists to single numbers (or small sets of numbers) and discard the primary data. The proposals being made in this paper

\footnotetext{
36 However it does not capture it completely: some species, for instance, may naturally be rare without being in danger of extinction.
} 
about the definition and assessment of biodiversity argue strongly against this practice; and (ii) these algorithms show very strong history-dependence. Which places we start with and the order in which the rules are applied make a very significant difference on the priority ordering of the set of places we work with. Consequently, if we introduce a cut-off above which we conserve places and below which we do not, the set of places targeted for conservation is similarly historydependent. While this initially appears to be a problem--or at least an undesirable feature--of our procedure, in some contexts we can convert it into a virtue. For instance, suppose that we have sociopolitical constraints that we must also try to satisfy while recommending a set of places for conservation (that is, a reserve network of some sort or other). We can initialize our procedure by choosing the first place differently and generate different sets of potentially conserved places which equally satisfy our biodiversity criteria. Then we can opt for the one that best satisfies our sociopolitical criteria. 


\section{Conclusions.}

We have succeeded in showing--possibly very unsatisfactorily for purists--how biodiversity may be defined and assessed. The solution of the assessment problem involved, in part, recourse to convention. This will probably be unpalatable--or, at least, undesirable--to some. I share this disquiet and await their less conventional alternative proposals. More importantly, the solution points to empirical work that must be done not solely for philosophical reasons, but for the practical goal of successfully conserving biodiversity. The empirical work that is critical in this manner is the identification of adequate estimator-surrogates. To the extent that philosophical analysis has had this practical scientific consequence--and one that has unfortunately not received adequate attention within science--it underscores the importance of philosophy for science.

Turning to the proposed solution of the problem of defining biodiversity, the following five points should be noted:

(i) the most interesting facet of this solution to the definition and assessment problems for biodiversity that it was only possible because of contextualization. We determined what biodiversity is by exploring what it was supposed to accomplish, and supposed to do so in the very specific and historically contingent context of the deterioration of our biological legacy during the past half-century. The rationale for this move was that this was the context in which the term biodiversity was introduced and gained currency. With contextualization, the solution appears easy, even trivial. Perhaps an important moral to be drawn from this story is that contextualization may be of general therapeutic value for the analysis of recalcitrant concepts; 
(ii) because a variety of prioritization algorithms have been proposed, what we have defined are a set of different concepts of biodiversity. However, there is one very good reason to suppose that these different definitions form a family (of closely related concepts): they all share the invocation of the complementarity and rarity rules. Moreover, if we restrict our definitions to algorithms that invoke only those rules, the differences between the definitions are relatively trivial. Some may prefer--or even demand--a single definition but it is hard to see any theoretical or practical rationale behind such a move;

(iii) the offered definitions are implicit rather than explicit since they are defined through the algorithms that are used, rather than by explicit necessary and sufficient conditions. It is incumbent upon those who prefer explicit definitions as a matter of principle to come up with a reasonable proposal for biodiversity. The difficulties discussed in Section 2 suggest--at the very least--that this will not be easy. Moreover, implicit definitions are de rigeur in science and mathematics and it does not seem reasonable to want to rule them illegitimate in conservation biology;

(iv) recalling the analogy between conservation biology and medicine that was drawn in Section 1, the solutions proffered here underscore the importance of a preventive rather than ameliorative approach in conservation biology. While the emphasis on rarity captures, in part, our concern for endangered species and other biological entities, complementarity takes us to all surrogates, no matter what their endangerment status is;

(v) the major flaw of the present analysis is that the treatment of the problem of endangered biological phenomena remains ad hoc. Places required for their 
protection were added to the list of prioritized places (in Section 4) by fiat. Further work needs to be done to see if this lacuna can be removed. 


\section{References}

Ackery, P. R. and Vane-Wright, R. I. 1984. Milkweed Butterflies. Ithaca, NY: Cornell University Press.

Aggarwal, A., Garson, J., Margules, C. R., Nicholls, A. O., and Sarkar, S. 2000. ResNet Manual. Ver 1.1. Report. Biodiversity and Biocultural Conservation Laboratory, University of Texas at Austin.

Angermeier, P. L. and Karr, J. R. 1994. "Biological Integrity versus Biological Diversity as Policy Directives." BioScience 44: 690 -697.

Bahadur, K. N. 1986. "Bamboos." In Hawkins, R. E., (ed.), Encycolpedia of Indian Natural History. New Delhi: Oxford University Press, pp. 30 -38.

Boyce, M. S. 1992. "Population Viability Analysis." Annual Review of Ecology and Systematics 23: $481-506$.

Brower, L. P. and Malcolm, S. B. 1991. "Animal Migrations: Endangered Phenomena." American Zoologist 31: 265 -276.

Buss, L. 1987. The Evolution of Individuality. Princeton: Princeton University Press.

Caughley, G. 1994. "Directions in Conservation Biology." Journal of Animal Ecology 63: $215-244$.

Caughley, G. and Gunn, A. 1996. Conservation Biology in Theory and Practice. Boston: Blackwell Science.

Crosby. 1986. Ecological Imperialism: the biological expansion of Europe, 900-1900. Cambridge: Cambridge University Press.

Eldredge, N. 1985. Unfinished Synthesis: Biological Hierarchies and Modern Evolutionary Thought. New York: Oxford University Press.

Ereshefsky, M. Ed. 1992. The Units of Evolution: Essays on the Nature of Species. Cambridge, MA: MIT Press.

Gaston, K. J. 1996. "Species Richness: Measure and Measurement." In Gaston, K. J., (ed.), Biodiversity: A Biology of Numbers and Difference. London: Blackwell, pp. $77-113$.

Guha, R. 1989. "Radical American Environmentalism and Wilderness Preservation: A Third World Critique." Environmental Ethics 11: 71 -83.

Guha, R. and Martinez-Alier, J. 1998. Varieties of Envirnomentalism: Essays North and South. New Delhi: Oxford University Press.

Holdridge, L. R. 1967. Life Zone Ecology. San Jose: Costa Rica.

Janzen, D. H. 1986. "The Future of Tropical Ecology." Annual Review of Ecology and Systematics 17(305 -324): 
Karr, J. R. 1991. "Biological Integrity: A Long Neglected Aspect of Water Resource Management." Ecological Applications 1: 66 -84.

Kirkpatrcik, J. B. 1983. "An Iterative Method for Establishing Priorities for the Selection of Nature Reserves: An Example from Tasmania." Biological Conservation 25: 127 134.

Leopold, A. 1949. A Sand County Almanac. Oxford, UK: Oxford University Press.

MacArthur, R. 1965. "Patterns of Species Diversity." Biological Review 40: 510 -533.

Magurran, A. E. 1988. Ecological Diversity and Its Measurement. Princeton: Princeton University Press.

Malpas, J. E. 1999. Place and Experience: A Philosophical Topography. Cambridge, UK: Cambridge University Press.

Margules, C. R., Nicholls, A. O., and Pressey, R. L. 1988. "Selecting Networks of Reserves to Maximize Biological Diversity." Biological Conservation 43: 63-76.

Maynard Smith, J. 1975. The Theory of Evolution. 3rd ed., Harmondsworth: Penguin.

Meffe, G. K. and Carroll, C. R. 1994. Principles of Conservation Biology. Sunderland, MA: Sinauer Associates.

Myers, N. 1988. "Threatened Biotas: "Hot-Spots" in Tropical Forests." Environmentalist 8: $187-208$.

Myers, N. 1990. "The Biodiversity Challenge: Expanded Hot-Spots Analysis." The Environmentalist 10(4): 243 -256.

Ohsawa, M. 1987. Life Zone Ecology of the Bhutan Himalaya. Chiba: Chiba University Laboratory of Ecology.

Primack, R. B. 1993. Essentials of Conservation Biology. Sunderland, MA: Sinauer.

Rebelo, A. G. and Siegfried, W. R. 1990. Biological Conservation 54: 15 -31.

Sarkar, S. 1998a. Genetics and Reductionism. New York: Cambridge University Press.

Sarkar, S. 1998b. "Restoring Wilderness or Reclaiming Forests?" Terra Nova 3(3): 35 -52.

Sarkar, S. and Margules, C. R. 2001. "Operationalizing Biodiversity for Conservation Planning." Submitted to Conservation Biology.

Shannon, C. E. 1948. "A Mathematical Theory of Communcation." Bell System Technical Journal 27: $379-423,623-656$.

Shrader-Frechette, K. S. and McCoy, E. D. 1993. Method in Ecology: Stragegies for Conservation. New York: Cambridge University Press.

Sokal, R. and Crovello, T. 1970. "The Biological Species Concept: A Critical Evaluation." American Naturalist 104: 127 -153. 
Soulé, M. E. 1985. "What is Conservation Biology?" BioScience 35: 727 -734.

Takacs, D. 1996. The Idea of Biodiversity: Philosophies of Paradise. Baltimore: Johns Hopkins Press.

Vane-Wright, R. I., Humphries, C. J., and Williams, P. H. 1991. "What to Protect?-Systematics and the Agony of Choice." Biological Conservation 55: 235 -254.

Williams, P. and Humphries, C. J. 1994. "Biodiversity, Taxonomic Relatedness, and Endemism in Conservation." In Forey, P. L., Humphries, C. J., and Vane-Wright, R. I., (ed.), Systematics and Conservation Evaluation. Oxford: Clarendon Press, pp. $269-287$.

Williams, P. H. and Humphreys, C. J. 1996. "Comparing Character Diversity among Biotas." In Gaston, K. J., (ed.), Biodiversity: A Biology of Numbers and Difference. Oxford: Blackwell Science, pp. 54 -76.

Wilson, E. O., (ed.) 1988. BioDiversity. Washington: National Academy Press. 